\title{
Rapid Progression of Lung Cancer Following Emergency Caesarean Section Led to Postpartum Acute Respiratory Failure
}

\author{
Tamami Watanabe ${ }^{1}$, Takeshi Yamashita ${ }^{1}$, Hitoshi Sugawara ${ }^{1}$, Takahiko Fukuchi ${ }^{1}$, Akira Ishii ${ }^{1}$, \\ Yoshiaki Nagai ${ }^{2}$, Fumiyoshi Ohyanagi ${ }^{2}$, Shinichiro Koyama ${ }^{2}$, Junko Ushijima ${ }^{3}$, \\ Kenjiro Takagi ${ }^{3}$ and Akira Tanaka ${ }^{4}$
}

\begin{abstract}
:
Our case patient was a 38-year-old pregnant Japanese woman who underwent emergency Caesarean section because of massive vaginal bleeding due to a low-lying placenta. Immediately after delivery, she presented with rapidly progressive dyspnea. Contrast-enhanced computed tomography revealed bilateral pleural effusion, lung nodules, multiple liver tumors, and multiple osteolytic lesions. Accordingly, epidermal growth factor receptor-mutant advanced lung adenocarcinoma was diagnosed. This report highlights the occurrence of rapid progression of lung cancer following delivery that led to postpartum acute respiratory failure, rather than due to pulmonary thromboembolism associated with the existing deep venous thrombosis of the inferior vena cava.
\end{abstract}

Key words: lung cancer combined with pregnancy, postpartum acute respiratory failure, epidermal growth factor receptor mutation, gefitinib

(Intern Med 58: 991-997, 2019)

(DOI: 10.2169/internalmedicine.1105-18)

\section{Introduction}

Critical conditions that require intensive care during the perinatal period are relatively rare (1). Acute respiratory failure after delivery has a broad differential diagnosis. Venous thromboembolism, cardiovascular disease, pulmonary hypertension, bronchial asthma, respiratory infection, neuromuscular disorders, and sepsis are recognized as non-obstetric causes of acute respiratory failure during pregnancy and puerperium (2), although lung cancer is a rare cause (3). In addition, rapid progression of lung cancer after delivery is uncommon.

We herein report a woman who developed a postpartum condition of dramatically deteriorating respiratory failure following emergency Caesarean section performed due to the rapid progression of epidermal growth factor receptor $(E G F R)$-mutant advanced lung adenocarcinoma, instead of pulmonary thromboembolism due to the existing deep venous thrombosis of the inferior vena cava.

\section{Case Report}

A 38-year-old gravida 2 para 1 pregnant Japanese woman developed difficulty walking due to pain in her right inguinal region and lower back at 35 weeks and 5 days of gestational age during her second pregnancy. Elective Caesarean section was accordingly scheduled because a low-lying placenta was detected by transvaginal echography at 36 weeks and 3 days of gestation. The patient was admitted to the De-

${ }^{1}$ Division of General Medicine, Department of Comprehensive Medicine 1, Saitama Medical Center, Jichi Medical University, Japan, ${ }^{2}$ Division of Pulmonary Medicine, Clinical Department of Internal Medicine, Saitama Medical Center, Jichi Medical University, Japan, ${ }^{3}$ Perinatal and Maternal Center, Department of Comprehensive Medicine 2, Saitama Medical Center, Jichi Medical University, Japan and ${ }^{4}$ Department of Pathology, Saitama Medical Center, Jichi Medical University, Japan

Received: February 27, 2018; Accepted: September 17, 2018; Advance Publication by J-STAGE: November 19, 2018

Correspondence to Dr. Hitoshi Sugawara, hsmdfacp@jichi.ac.jp 


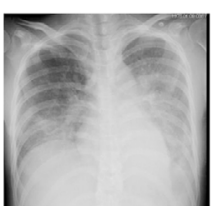

Day 8

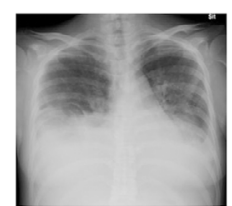

Day 13

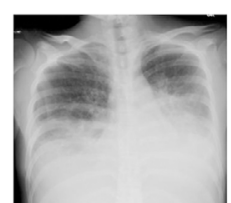

Day 17

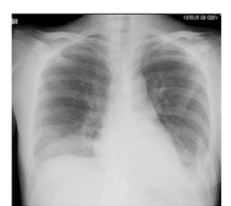

Day 24

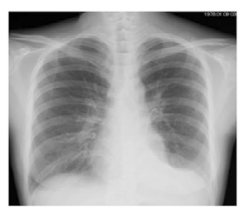

Day 43

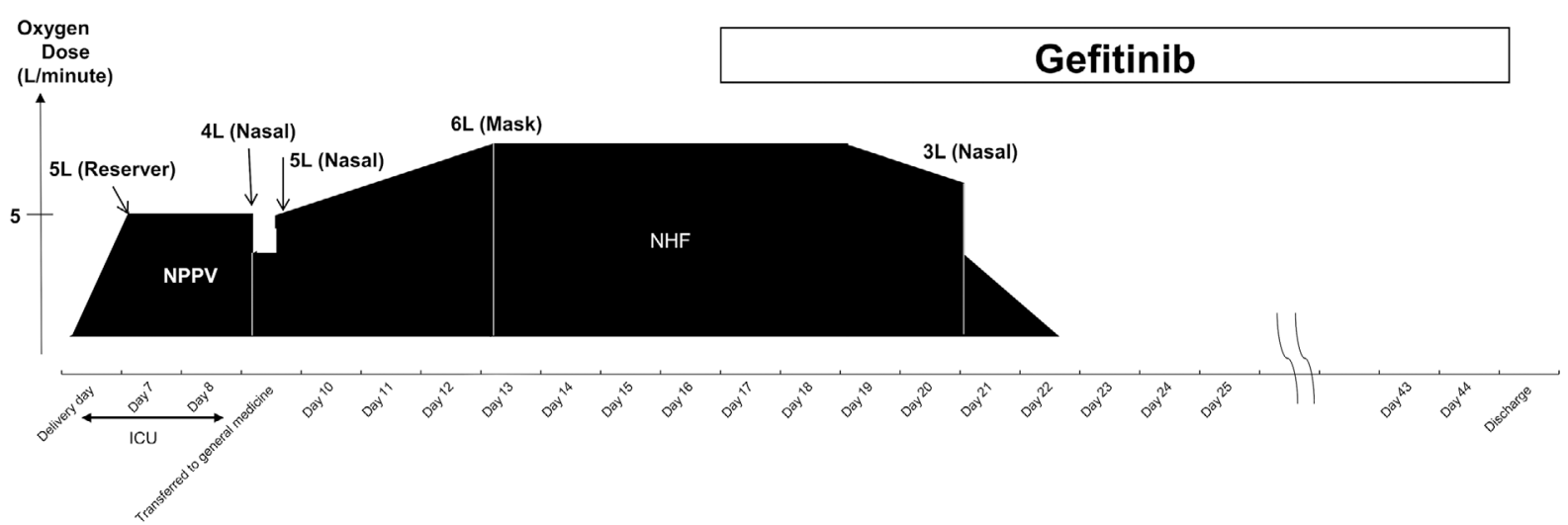

Figure 1. The clinical course showing the chest X-ray findings, treatment regimens, and the requirement and modalities of oxygen therapy. ICU: intensive-care unit, NHF: nasal high-flow oxygen, NPPV: non-invasive positive-pressure ventilation

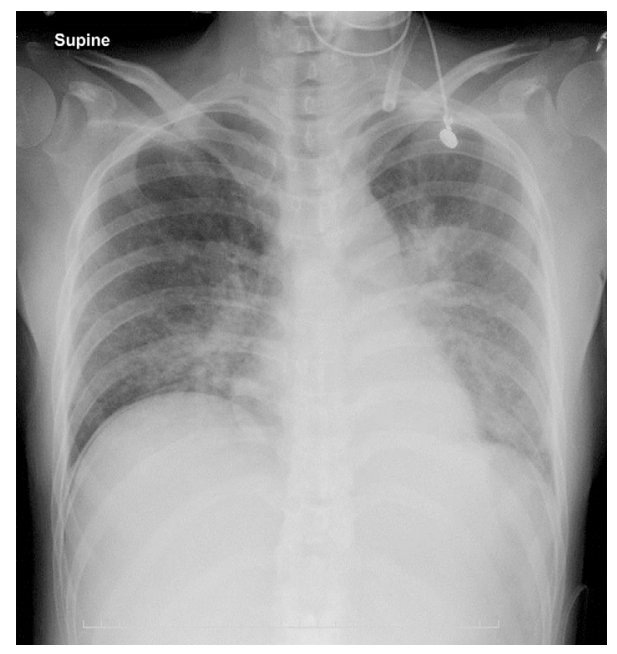

Figure 2. Chest X-ray findings on hospital day 6 after delivery by Caesarean section showing pulmonary congestion and reduced permeability, primarily in the lower lung fields.

partment of Obstetrics. Before this operation, her body temperature was $36.3^{\circ} \mathrm{C}$, pulse rate was 83 beats/min and regular, blood pressure was $116 / 50 \mathrm{mmHg}$, respiratory rate was 15 breaths/min, and percutaneous arterial blood oxygen saturation $\left(\mathrm{SpO}_{2}\right)$ was $98 \%$ on room air. Furthermore, her physical findings remained unchanged, expect for the lower abdominal pain. On hospital day (HD) 6 (i.e., 37 weeks and 1 day gestation), the patient developed massive vaginal bleeding and underwent emergency Caesarean section for the same. She was then transferred to the intensive-care unit (ICU) because of dramatically deteriorating dyspnea.

The patient's clinical course after emergency Caesarean section is depicted in Fig. 1. On HD 6 after delivery [post- operative day (POD) 0], chest X-ray (Fig. 2) revealed cardiac dilatation and opacity, predominantly in the bilateral lower lung fields. Progression of anemia from hemoglobin $(\mathrm{Hb})$ level $10.1 \mathrm{~g} / \mathrm{dL}$ prior to childbirth to $6.1 \mathrm{~g} / \mathrm{dL}$ after was noted. Contrast-enhanced whole-body computed tomography on HD 7 (POD 1) showed bilateral pleural effusions, multiple lung nodules (Fig. 3A), multiple liver masses (Fig. 3B), and multiple osteolytic changes (Fig. 3C). There were no signs of pulmonary embolism; however, thrombi were detected in the splenic vein, abdominal inferior vena cava, and the left common iliac vein (Fig. 3C).

On the same day, her $\mathrm{SpO}_{2}$ had deteriorated to $94 \%$ with $5 \mathrm{~L} / \mathrm{min}$ (LPM) oxygen reservoir mask administration, and non-invasive positive-pressure ventilation was administered along with a diuretic, which led to the resolution of respiratory distress. On HD 9, the patient was transferred to the Division of General Medicine for further treatment.

On examination in our division, the patient was conscious and well-oriented but reported left hip pain. Her body temperature was $37.1{ }^{\circ} \mathrm{C}$, pulse rate was 93 beats/min and regular, blood pressure was $126 / 82 \mathrm{mmHg}$, respiratory rate was 25 breaths/min, and $\mathrm{SpO}_{2}$ was $96 \%\left(\mathrm{O}_{2}: 5 \mathrm{~L} /\right.$ nasal cannula). The conjunctiva was anemic and icteric. Several rubbery hard lymph nodes (1-2 cm in size) were palpated on the left side of the neck and fovea superior to the left clavicle. The respiratory sounds were attenuated in the bilateral lower lung areas without rales. Bilateral breast engorgement and a midline Caesarean operation scar were observed on the lower abdomen. The remainder of the examination was unremarkable.

Notable laboratory findings included the following: white blood cell count $(11,470 / \mu \mathrm{L})$, normocytic normochromic 


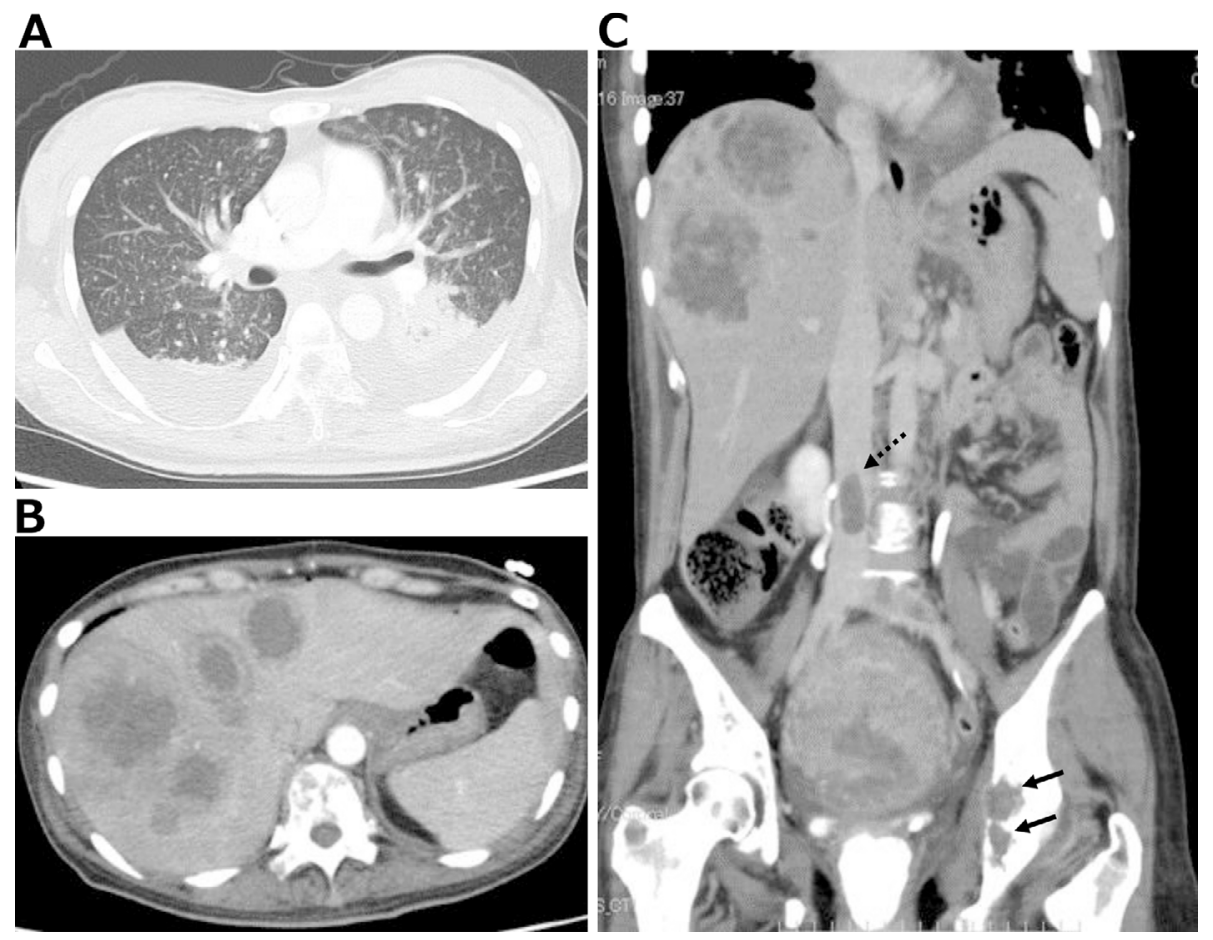

Figure 3. Contrast-enhanced whole-body computed tomography images showing bilateral pleural effusion, multiple lung small nodules (A), multiple liver tumors (B), and osteolytic changes in the iliac bones (C: black solid arrows). No signs of pulmonary embolism can be seen; however, thrombi can be seen in the splenic vein, abdominal inferior vena cava ( $C$ : black dotted arrow), and the left common iliac vein.

A

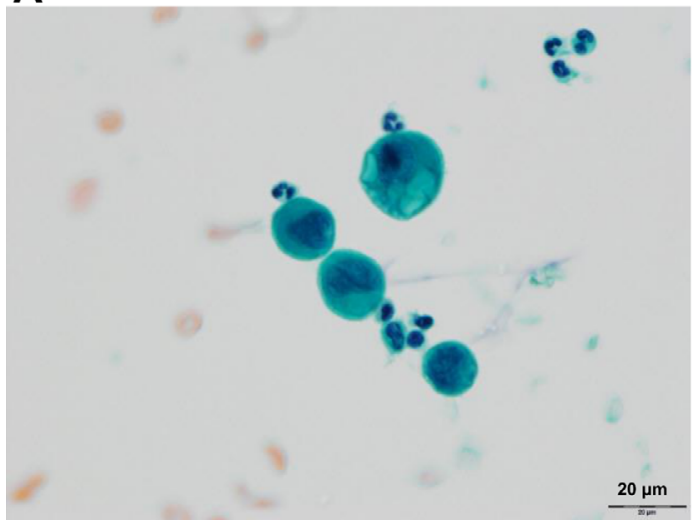

B

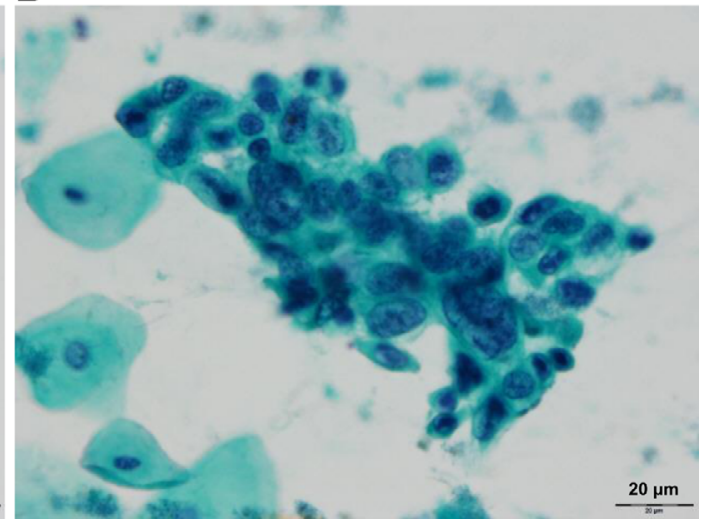

Figure 4. Sputum cytology of Papanicolaou stain (A; original magnification, 20x) indicating adenocarcinoma cells with nuclear ubiquity, nuclear enlargement, and nuclear irregularity. Cytology of pleural effusion of Papanicolaou stain ( $B$; original magnification, 20x) showing adenocarcinoma with an abnormal cell cluster, indicating nuclear enlargement and anisokaryosis.

anemia ( $\mathrm{Hb}, 7.7 \mathrm{~g} / \mathrm{dL})$, hypoalbuminemia $(2.1 \mathrm{~g} / \mathrm{dL})$, and elevated levels of lactate dehydrogenase $(4,467$ units/L), alkaline phosphatase $(1,651$ units/L), and tumor markers (carcinoembryonic antigen, $44.0 \mathrm{ng} / \mathrm{dL}$; carbohydrate antigen 19-9, 154.7 U/mL; carbohydrate antigen 125, $243.6 \mathrm{U} / \mathrm{mL}$; and carbohydrate antigen 15-3, $55 \mathrm{U} / \mathrm{mL})$.

Pleural fluid (Fig. 4A) and sputum (Fig. 4B) cytology revealed poorly differentiated adenocarcinoma. However, detection of the primary lesions in the lungs was difficult due to massive pleural effusion. Findings of upper gastrointestinal endoscopy, colonoscopy, and breast ultrasonography were unremarkable for confirming the primary lesion as adenocarcinoma. The results of a pathological examination of her placenta were normal.

The patient stopped breastfeeding after the colostrum stage. Due to worsening of her respiratory condition on HD 13 , oxygen therapy was changed to nasal high-flow oxygen (50\%; $50 \mathrm{~L} / \mathrm{min})$. 
A

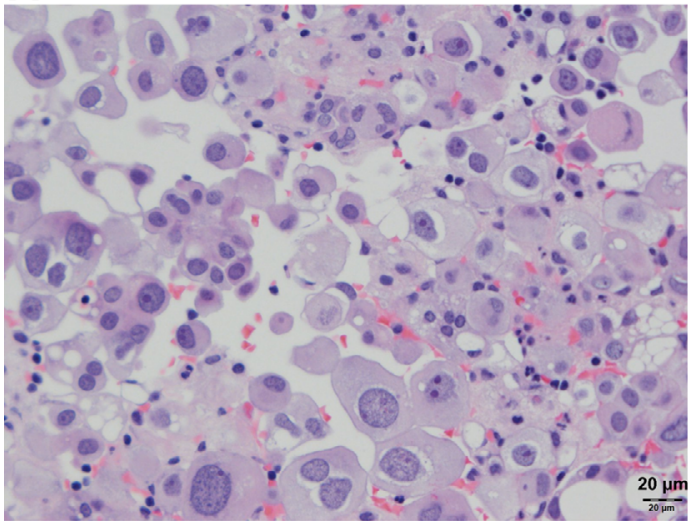

B

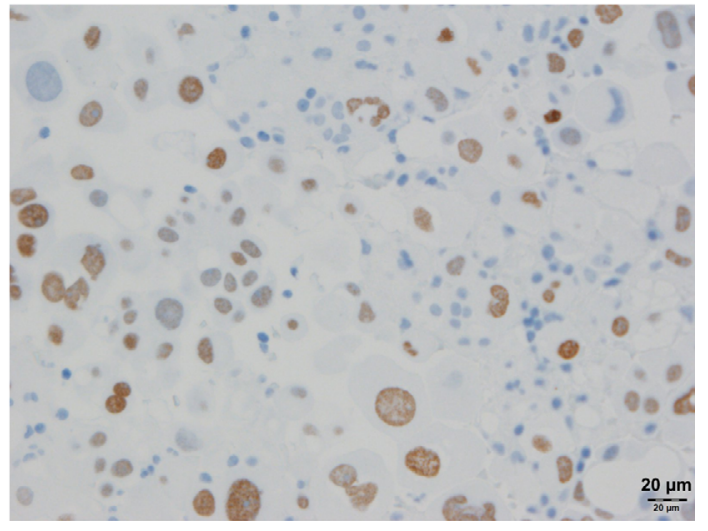

Figure 5. Cell block cytology of the pleural effusion. Hematoxylin and Eosin staining (A; original magnification, 20x) demonstrated adenocarcinoma with abnormal cells showing nuclear enlargement and anisokaryosis; immunostaining for thyroid transcription factor-1 (TTF-1, B; original magnification, 20x) showed positive expression in the nuclei of abnormal cells.

Cell block cytology of the pleural effusion on HD 13 revealed adenocarcinoma (Fig. 5A). Results of immunostaining were positive for cytokeratin $(\mathrm{CK}) 7$, thyroid transcription factor-1 (Fig. 5B), and napsin A but negative for CK20. These findings were consistent with those of lung adenocarcinoma. In addition, estrogen receptor (ER) was $1-5 \%$ positive. Owing to the detection of the EGFR mutation (deletion of E746-A750 in exon 19) by peptide nucleic acid-locked nucleic acid polymerase chain reaction clamp method on HD 17, $250 \mathrm{mg} /$ day gefitinib (a selective inhibitor of the tyrosine kinase domain of EGFR) therapy was initiated on the same day. On HD 20, the patient was transferred to the Division of Respiratory Medicine.

After the initiation of gefitinib therapy, pleural effusion considerably reduced, and the $\mathrm{SpO}_{2}$ gradually improved. Accordingly, the patient was discharged along with her baby on HD 45 and continued treatment on an outpatient basis without using analgesics and even became ambulatory.

\section{Discussion}

This report highlights the fact that postpartum acute respiratory failure following emergency Caesarean section may not be caused by pulmonary thromboembolism, despite the presence of deep venous thrombosis in the inferior vena cava; instead, it may result from rapid progression following delivery of EGFR-mutant advanced lung adenocarcinoma, although advanced lung adenocarcinoma is rarely encountered during pregnancy.

The clinical course of this patient highlights five important clinical issues: (i) advanced lung cancer can be a cause of postpartum acute respiratory failure; (ii) the reason for the delay in the diagnosis of lung cancer combined with pregnancy needs to be clarified; (iii) the reason for the aggravation of symptoms related to lung cancer after delivery needs to be clarified; iv) if any gene biomarker is positive, ascertaining whether or not an appropriate molecular- targeted drug can be used to save the life of a pregnant woman with advanced lung cancer despite a poor performance status is important; and (v) the safety of biomarkerspecific medicines, particularly gefitinib and other EGFR tyrosine kinase inhibitors (EGFR-TKIs) during the breastfeeding period remains unclear.

Pulmonary thromboembolism is complicated in $0.05 \%$ $0.3 \%$ of pregnancies and is the leading nonobstetric cause of acute respiratory failure in pregnant patients (2). The incidence of malignant tumors in pregnant women was reported to be 1 in 1,000 pregnant women; although a relatively high incidence of breast cancer, cervical cancer, malignant lymphoma, malignant melanoma, and leukemia is reported in pregnant women, the occurrence of lung cancer is extremely rare (3). The most common histological type of lung cancer is non-small cell lung cancer, accounting for $85 \%$ of all lung cancers during pregnancy (4). Physicians typically do not expect malignant tumors, particularly lung cancer, in pregnant women due to its rarity. However, $>80 \%$ of all such diagnosed cases have advanced stage III or stage IV lung cancer (5), which is associated with a poor prognosis (6).

Symptoms of lung cancer, such as respiratory distress and fatigue, tend to be perceived as symptoms related to pregnancy, which may explain the delayed diagnosis of lung cancer in pregnant women (7). Another possible reason is the tendency for women to avoid undergoing radiographic examinations during pregnancy (8).

The increased secretion of estrogen in pregnancy is likely to be the primary reason for the rapid tumor growth during this period. In approximately $50 \%$ of all patients with pulmonary adenocarcinoma, tumor cells show the positive expression of ER (9). In addition, estradiol promotes the proliferation of ER-positive lung cancer cells (10). In the present case, the $1-5 \%$ positivity for ER expression may have contributed to the tumor growth. The activation of other tumor growth pathways, such as epidermal growth factor, 


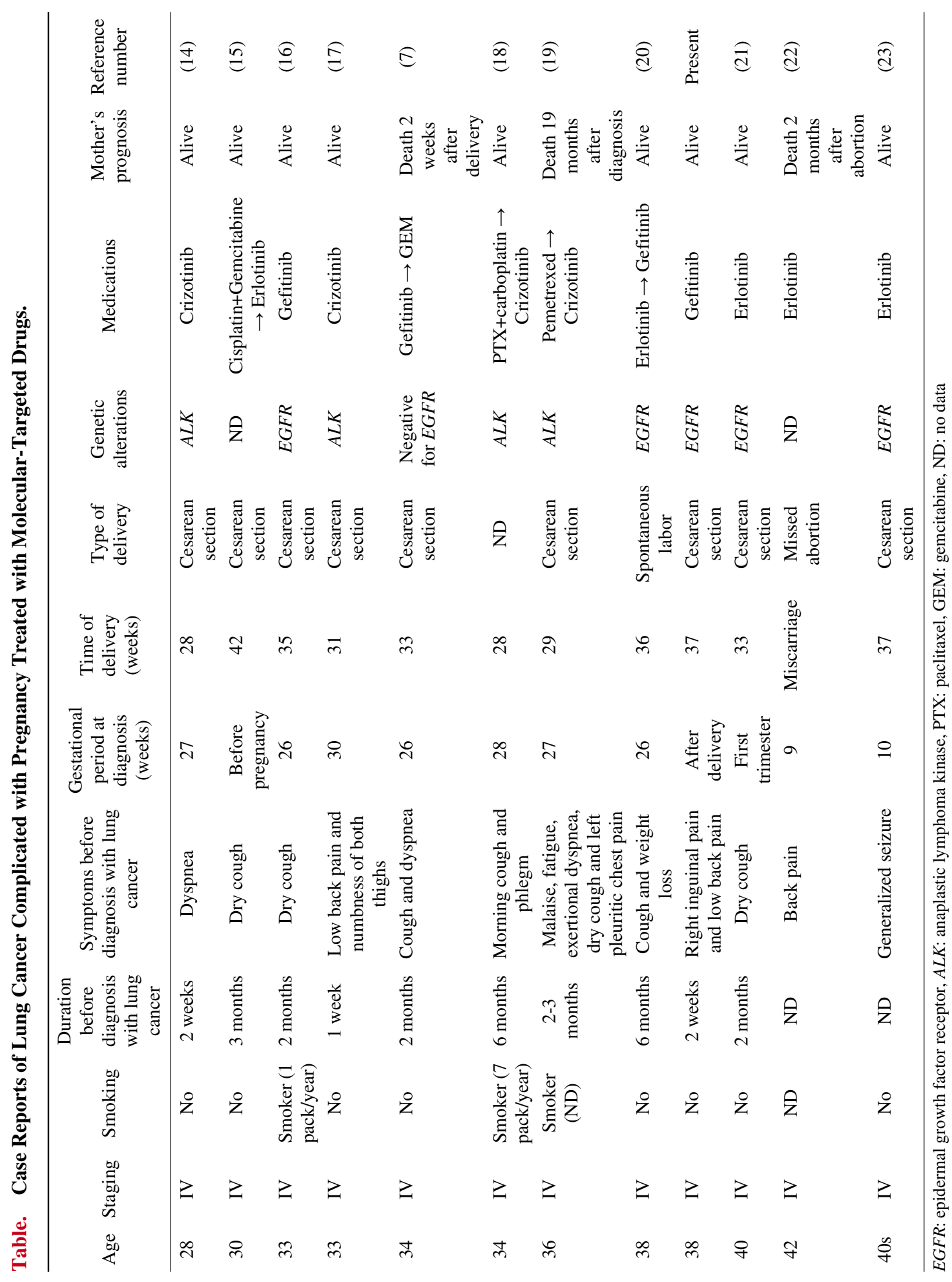

transforming growth factor (TGF)- $\alpha$, and insulin-like growth factors by estrogen, is another potential mechanism underlying the rapid tumor growth (11).

The following immune escape mechanisms are reportedly shared between malignant tumors and the fetoplacental function (12): i) cytotoxic T lymphocytes (CTLs) do not recognize tumor histocompatibility complex (major histocompatibility complex) antigens, i.e., human leukocyte antigen-G, thereby inhibiting the natural killer cell activity; ii) indoleamine 2,3-dioxygenase produced by macrophages inhibits

CTL activity; iii) the secretion of inhibitory cytokines (particularly IL-6, IL-10, vascular endothelial growth factor, granulocyte-colony stimulating factor, and TGF- $\beta$ ) shifts to Th2-type cytokine secretion; and iv) regulatory $\mathrm{T}$ cells express the transcription factor Foxp3 and induce immune tolerance. Each of these mechanisms is observed in fetal placenta and malignant tumors, and immunological tolerance during pregnancy further inhibits immune responses. However, immediately after delivery, the immune escape mechanism is terminated, which is believed to lead to the sudden 
growth of the tumor.

Acute exacerbation after childbirth is likely attributable to termination of immunological tolerance upon childbirth. Restoration of the immune response in such a situation may induce an excessive immune response to the tumor and the rapid progression of the disease following childbirth. Furthermore, increased cytokine secretion induced by tissue damage as a result of Caesarean section may have contributed to the acute exacerbation of the tumor in this case, as highlighted in the report of a patient who experienced a similar clinical course (13).

We reviewed the literature for reports pertaining to lung cancer in pregnant women treated with molecular-targeted drugs (Table) (7, 14-23) using the PubMed database, searching for articles published in English and Japanese as of May 2017 for the following keywords: "lung cancer," "pregnancy," and "molecular-target drug (gefitinib, erlotinib or crizotinib)." In addition, we searched the Igaku Chuo Zasshi (ICHUSHI) database, which is a Japanese document repository, for only those articles wherein molecular-targeted drugs were used for the treatment of lung cancer combined with pregnancy. The age range of the 12 patients (including the present case) was 28-42 years, and 8 were non-smokers. All patients had stage IV lung cancer at the diagnosis. Several months passed before a diagnosis was obtained for seven patients. Symptoms prior to the diagnosis commonly included respiratory symptoms, such as cough and respiratory distress. The timing of the diagnosis tended to be during the second trimester; in most cases, the mode of delivery was Caesarean section, the timing of which was determined according to the mother's general condition. In patients who were gene mutation-positive, the prognosis tended to be good despite the advanced stage of cancer, although it cannot be denied that a publication bias exists. The availability of targeted therapy based on a specific biomarker profile offers a considerable benefit and an increased chance to achieve remission, as observed in our patient.

To our knowledge, there are no data pertaining to the excretion of EGFR-TKIs in human milk and its potential effects on a breastfed infant or on milk production. The safety of gefitinib and other EGFR-TKIs during the breastfeeding period has not yet been established in humans (24-27). Animal studies indicate that gefitinib (24) and afatinib (25) are present in rat milk at a concentration higher than that in maternal plasma. The administration of osimertinib (26) to rats during gestation and early lactation has been associated with adverse effects, including reduced growth rate and neonatal death. Based on these findings, we advised the present patient to discontinue breastfeeding while receiving gefitinib therapy in order to ensure the safety of her baby.

In conclusion, physicians should be aware of the possibility that rapid progression of advanced lung cancer rather than pulmonary thromboembolism, as is generally expected in such cases, may cause postpartum acute respiratory failure, even when deep venous thrombosis exists in the inferior vena cava. We believe that it is possible to prolong a pa- tient's life, even in the setting of life-threatening advanced lung cancer combined with pregnancy, in the era of molecular-targeted therapy and biomarker-specific medicines for malignancies.

The outline of the present case was reported at the eighth Annual Conference of Japan Primary Care Association (May 14, 2017, Takamatsu).

\section{The authors state that they have no Conflict of Interest (COI).}

\section{Acknowledgement}

We appreciate the expert technical support by Tetsuya Kawano, a cytotechnologist at the Department of Pathology, Saitama Medical Center, Jichi Medical University.

\section{References}

1. Rush B, Martinka P, Kilb B, McDermid RC, Boyd JH, Celi LA. Acute respiratory distress syndrome in pregnant women. Obstet Gynecol 129: 530-535, 2017.

2. Bhatia PK, Biyani G, Mohammed S, Sethi P, Bihani P. Acute respiratory failure and mechanical ventilation in pregnant patient: A narrative review of literature. J Anaesthesiol Clin Pharmacol 32: 431-439, 2016.

3. Pavlidis NA. Coexistence of pregnancy and malignancy. Oncologist 7: 279-287, 2002.

4. Mitrou S, Petrakis D, Fotopoulos G, Zarkavelis G, Pavlidis N. Lung cancer during pregnancy: a narrative review. J Adv Res 7: 571-574, 2016.

5. Jackisch C, Louwen F, Schwenkhagen A, et al. Lung cancer during pregnancy involving the products of conception and a review of the literature. Arch Gynecol Obstet 268: 69-77, 2003.

6. Pentheroudakis G, Pavlidis N. Cancer and pregnancy: poena magna, not anymore. Eur J Cancer 42: 126-140, 2006.

7. Hata A, Harada Y, Seo R, et al. A case of lung cancer combined with pregnancy; dramatically deteriorating condition after caesarian section. Nihon Kokyuki Gakkai Zasshi (J Jpn Respir Soc) 47: 585-590, 2009 (in Japanese, Abstract in English).

8. Mujaibel K, Benjamin A, Delisle M, Williams K. Lung cancer in pregnancy: case reports and review of the literature. J Matern Fetal Med 10: 426-432, 2001.

9. Hayama M, Chida M, Tamura M, Kobayashi S, Oyaizu T, Honma $\mathrm{K}$. Unexpected rapid growth of estrogen receptor positive lung cancer during pregnancy. Ann Thorac Cardiovasc Surg 47: 8-11, 2013.

10. Nose N, Sugio K, Oyama T, et al. Association between estrogen receptor-beta expression and epidermal growth factor receptor mutation in the postoperative prognosis of adenocarcinoma of the lung. J Clin Oncol 27: 411-417, 2009.

11. Ignar-Trowbridge DM, Pimentel M, Parker MG, McLachlan JA, Korach KS. Peptide growth factor cross-talk with the estrogen receptor requires the $\mathrm{A} / \mathrm{B}$ domain and occurs independently of protein kinase C or estradiol. Endocrinology 137: 1735-1744, 1996.

12. Hayakawa $S$. No cancer in cancers: evolutionary trade-off between successful viviparity and tumor escape from the adaptive immune system. Med Hypotheses 66: 888-897, 2006.

13. But Hadzic J, Secerov A, Zwitter M, et al. Metastatic adenocarcinoma of the lung in a 27 -year-old pregnant woman. J Thorac Oncol 2: 450-452, 2007.

14. Sugino K, Yasuda M, Kimishima S, et al. A case of lung cancer complicating pregnancy. Sanka to Fujinka 83: 447-450, 2016 (in Japanese).

15. Zambelli A, Prada GA, Fregoni V, Ponchio L, Sagrada P, Pavesi 
L. Erlotinib administration for advanced non-small cell lung cancer during the first 2 months of unrecognized pregnancy. Lung Cancer 60: 455-457, 2008.

16. Gil S, Goetgheluck J, Paci A, et al. Efficacy and safety of gefitinib during pregnancy: case report and literature review. Lung Cancer 85: 481-484, 2014

17. Hirose $Y$, Doi S, Orisaka M, et al. A case of lung cancer complicating pregnancy with placental metastasis. Sanfujinka No Jissai (Pract Gynecol Obstet) 65: 347-352, 2016 (in Japanese).

18. Sarman N, Levent E, Yener NA, Örki A, Sayg1 A. Lung cancer and pregnancy. Lung Cancer 79: 321-323, 2013.

19. Neves I, Mota PC, Hespanhol VP. Lung cancer during pregnancy: an unusual case. Rev Port Pneumol 20: 46-49, 2014.

20. Lee CH, Liam CK, Pang YK, Chua KT, Lim BK, Lai NL. Successful pregnancy with epidermal growth factor receptor tyrosine kinase inhibitor treatment of metastatic lung adenocarcinoma presenting with respiratory failure. Lung Cancer 74: 349-351, 2011.

21. Rivas G, Llinás N, Bonilla C, Rubiano J, Cuello J, Arango N. Use of erlotinib throughout pregnancy: a case-report of a patient with metastatic lung adenocarcinoma. Lung Cancer 77: 469-472, 2012.

22. Boussios S, Han SN, Fruscio R, et al. Lung cancer in pregnancy: report of nine cases from an international collaborative study. Lung Cancer 82: 499-505, 2013.

23. Ji Y, Schwartz J, Hartford A, Ramsey J, Phillips J, Verschraegen
C. Successful treatment of non-small cell lung cancer with erlotinib throughout pregnancy. JAMA Oncol 1: 838-840, 2015.

24. Highlights of prescribing iformation of IRESSA ${ }^{\mathrm{TM}}$ (gefitinib) tablets [Internet]. [cited 2018 April 29]. Available from: https://www.a ccessdata.fda.gov/drugsatfda_docs/label/2015/206995s000lbl.pdf

25. Highlights of prescribing iformation of GILOTRIFTM (afatinib) tablets [Internet]. [cited 2018 April 29]. Available from: https://ww w.accessdata.fda.gov/drugsatfda_docs/label/2013/201292s000lbl.pd

26. Highlights of prescribing iformation of TAGRISSO ${ }^{\mathrm{TM}}$ (osimertinib) tablet [Internet]. [cited 2018 April 29]. Available from: http s://www.accessdata.fda.gov/drugsatfda_docs/label/2017/208065s00 6lbl.pdf

27. Highlights of prescribing iformation of TARCEVA ${ }^{\mathrm{TM}}$ (erlotinib) tablet [Internet]. [cited 2018 April 29]. Available from: https://ww w.accessdata.fda.gov/drugsatfda_docs/label/2016/021743s025lbl.pd

The Internal Medicine is an Open Access journal distributed under the Creative Commons Attribution-NonCommercial-NoDerivatives 4.0 International License. To view the details of this license, please visit (https://creativecommons.org/licenses/ by-nc-nd/4.0/).

(C) 2019 The Japanese Society of Internal Medicine Intern Med 58: 991-997, 2019 